LBNL-49483

CBP tech note 411

\title{
Beam Break Up Analysis for the Berkeley Recirculating Linac Light Source
}

\author{
S. DE SANTIS AND A. ZHOLENTS \\ Ernest Orlando Lawrence Berkeley National Laboratory \\ One Cyclotron Road \\ Berkeley, California 94720, USA
}

\section{INTRODUCTION}

We describe here a study of a single bunch Beam Break Up (BBU) instability for a proposed Berkeley fast x-ray facility based on a recirculating linac [1]. The recirculating linac employs a $600 \mathrm{MeV}$ superconducting RF linear accelerator and the electron beam energy of $\sim 2.5 \mathrm{GeV}$ is reached over four beam passes through the linac. A $120 \mathrm{MeV}$ superconducting RF linear accelerator is used as an injector to the recirculating linac. The machine parameters are listed in Appendix A.

The equation describing the transverse displacement $x(s, z)$ of the electrons in an accelerated bunch, as a function of their longitudinal position within the bunch $z$, can be written in the form [2]:

$$
\frac{d}{d s}\left[\gamma(s) \frac{d x}{d s}\right]+k^{2}(s) \gamma(s) x(s, z)=r_{0} \int_{z}^{\infty} \rho\left(z^{\prime}\right) W_{\perp}\left(z^{\prime}-z\right) x\left(s, z^{\prime}\right) d z^{\prime}
$$

where $\gamma$ is the relativistic factor, $k$ the focussing strength, $r_{0}$ the classical electron radius, $\rho$ the bunch density, $W_{\perp}$ the transverse wake function per unit length and $s$ indicates the position along the linac. We assume infinitesimally small transverse beam dimensions (a good approximation, when the bunch dimensions are much less than the size of the beam pipe), so that $x$ has to be interpreted as the displacement of the centre of a bunch slice. We also assume a bunch length much less than the betatron wavelength, therefore the displacement in the RHS of Eq.(1) is not retarded and, finally, we use an average transverse wake function obtained averaging the calculated short range wake of a single cavity [2] over the linac length.

At first we consider the effect associated with a displacement of the electron bunch at the injection into the perfectly aligned linac. Later we extend the analysis to the case of misalignments of the linac RF cavities and cryomodules. 
Throughout the paper we compare the results obtained to the output of a simple tracking code, written as a Mathematica notebook (see Appendix B).

In order to keep the analytical expressions reasonably simple, we model the linac length as entirely filled with RF cavities (thus neglecting all the drift spaces, accounting for as much as one third of the total length). This causes the wakefield intensity used in the analysis to be bigger than the actual value and the average accelerating gradient to be smaller. It is shown in the paper than this corresponds to a conservative estimate of the BBU growth.

\section{ZERO FOCUSSING}

If there are no focussing elements on the recirculated accelerating section, as is the case in the Berkeley linac, then $\mathrm{k}(\mathrm{s}) \equiv 0$ and we can rewrite Eq.(1) as:

$$
\frac{d^{2} x}{d u^{2}}+\frac{1}{u} \frac{d x}{d u}=\frac{r_{0}}{\gamma_{i} G^{2} u} \int_{z}^{\infty} \rho\left(z^{\prime}\right) W_{\perp}\left(z^{\prime}-z\right) x\left(u, z^{\prime}\right) d z^{\prime}
$$

where $u=1+G s$ is a new variable, defined with the further assumption of a uniform

acceleration over the entire linac length $L, \gamma(s)=\gamma_{i}(1+G s)$ and $G=\left(\gamma_{f} / \gamma_{i}-1\right) / L \cdot \gamma_{i}$ and $\gamma_{f}$ are the energy at the beginning and at the end of each pass respectively.

We can solve Eq.(2) using a perturbation technique, beginning with a first order solution $x_{(1)}:$

$$
\frac{d^{2} x_{(1)}}{d u^{2}}+\frac{1}{u} \frac{d x_{(1)}}{d u}=\frac{r_{0}}{\gamma_{i} G^{2} u} \int_{z}^{\infty} \rho\left(z^{\prime}\right) W_{\perp}\left(z^{\prime}-z\right) x_{0} d z^{\prime}
$$

with initial conditions: $x(s=0)=x_{0}, x^{\prime}(s=0)=x_{0}^{\prime}$ for all the electrons in the bunch. This yields:

$$
x_{(1)}(z)=x_{0}+A_{0}(z)[u-1-\ln u]+x_{0}^{\prime} L \frac{\gamma_{i}}{\Delta \gamma} \ln u
$$

where

$$
A_{0}(z)=x_{0} \frac{r_{0}}{\gamma_{i} G^{2}} \int_{z}^{\infty} \rho\left(z^{\prime}\right) W_{\perp}\left(z^{\prime}-z\right) d z^{\prime}
$$

Therefore, the relative increment of the transverse displacement at the end of the linac $(s=L)$ is:

$$
\frac{x_{(1)}}{x_{0}}=1+\frac{A_{0}}{x_{0}}[G \cdot L-\ln (1+G \cdot L)]+\frac{x_{0}^{\prime}}{x_{0}} L \frac{\gamma_{i}}{\Delta \gamma} \ln (1+G \cdot L)
$$

It is convenient to examine in detail the cases of coordinate and angular displacement at injection separately. For the numerical results the wake for the TESLA RF cavities [3] and a 
gaussian bunch $(\sigma=1.5 \mathrm{~mm})$ are considered, but the formulas obtained below are valid for an arbitrary bunch distribution.

2.1Coordinate displacement and no angular displacement: $x(s=0, z)=x_{0}, x^{\prime}(s=0, z)=0$.

If we assume no angle error at injection Eq.(6) becomes:

$$
\frac{x_{(1)}}{x_{0}}=1+\frac{A_{0}}{x_{0}}[G \cdot L-\ln (1+G \cdot L)]
$$

and $x_{(1)}$ can be easily calculated.

Figure 1 shows the relative displacements, $x_{(1)} / x_{0}-1$, along the bunch at the end of each pass of the linac. The results from our tracking code are also reported.

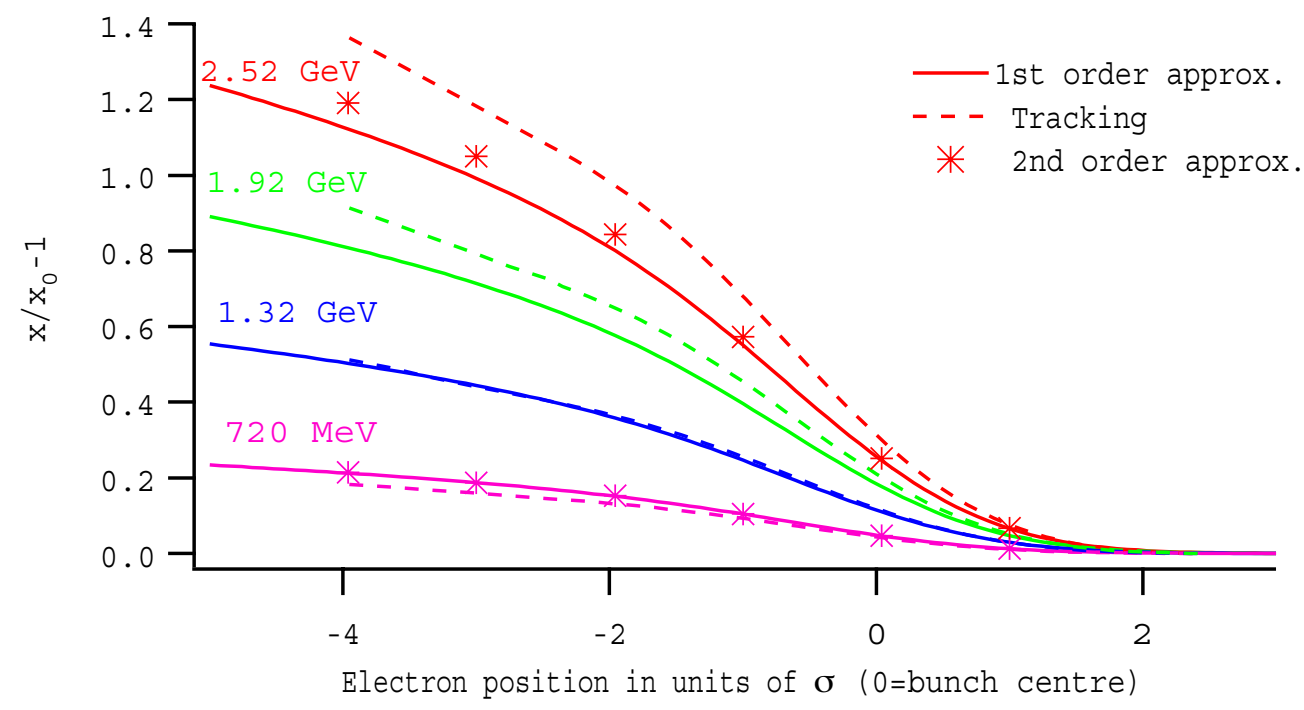

Figure 1. Cumulative transverse displacement (relative to the bunch head), as a function of the electron position within the bunch, after each pass.

We see that after the first pass the bunch tail is displaced by as much as $20 \%$ of its initial displacement at injection more than the bunch head. Since this value is not negligible, let us obtain the second order solution of Eq.(2) to check that the actual increase is not much different:

$$
\frac{d^{2} x_{(2)}}{d u^{2}}+\frac{1}{u} \frac{d x_{(2)}}{d u}=\frac{r_{0}}{\gamma_{i} G^{2} u} \int_{z}^{\infty} \rho\left(z^{\prime}\right) W_{\perp}\left(z^{\prime}-z\right) x_{(1)} d z^{\prime}
$$

Replacing Eq.(7) in Eq.(8) we obtain:

$$
\frac{d^{2} x_{(2)}}{d u^{2}}+\frac{1}{u} \frac{d x_{(2)}}{d u}=\frac{A_{0}}{u}+A_{1}\left(1-\frac{1}{u}-\frac{\ln u}{u}\right)
$$


where we have indicated

$$
A_{1}(z)=x_{0}\left(\frac{r_{0}}{\gamma_{i} G^{2}}\right)^{2} \int_{0}^{\infty} \rho\left(z^{\prime}+z\right) W_{\perp}\left(z^{\prime}\right) \int_{0}^{\infty} \rho\left(\zeta+z^{\prime}+z\right) W_{\perp}(\zeta) d \zeta d z^{\prime}
$$

The solution of Eq.(9), at the end of the linac $(u=1+G L)$, is

$$
\frac{x_{(2)}}{x_{0}}=\frac{x_{(1)}}{x_{0}}+\frac{A_{1}}{x_{0}}\left[\frac{3}{2} G \cdot L+\frac{1}{4} G^{2} L^{2}-\left(\frac{3}{2}+G \cdot L\right) \ln (1+G \cdot L)\right]
$$

Comparing Eqs.(5) and (10), we see that $A_{1}<A_{0}$, if $A_{0}<1$. Moreover, the term in square brackets in Eq.(11) is smaller than the analogous term in Eq.(7), for small enough accelerating gradients $G$. All these conditions are verified in the case we are interested in. After numerically evaluating Eq.(11) at several positions along the bunch, shown by stars in Fig. 1, we conclude that we can safely use the first order approximation only. Since later passes are equivalent to having a longer linac, we see that the second order approximation at the highest energy begins to be somewhat different from the first order result. In the next Section we will study this in a greater detail.

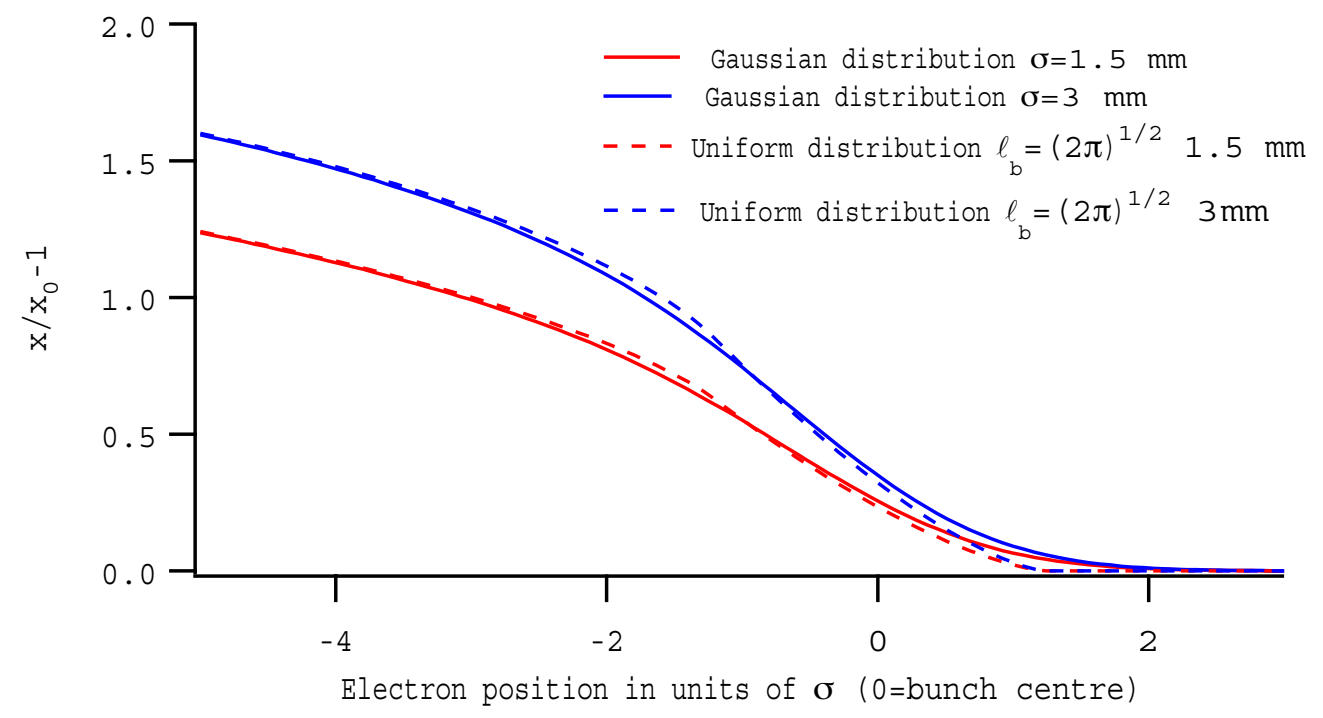

Figure 2. Displacement at the end of four passes for different bunch lengths and distributions.

Figure 2 shows the transverse displacement at the end of the four linac passes for two different bunch lengths $(1.5$ and $3 \mathrm{~mm})$ and two different charge distributions (gaussian and uniform). There are no apparent differences between the two distribution, while the effect of the bunch length is instead substantial. 
2.2 Angular displacement and no coordinate displacement: $x(s=0, z)=0, x^{\prime}(s=0, z)=x_{0}^{\prime}$.

In this case Eq.(4) becomes:

$$
x_{(1)}(z)=x_{0}^{\prime} L \frac{\gamma_{i}}{\Delta \gamma} \ln u
$$

This, as opposed to Eq.(7) doesn't depend on $\mathrm{z}$ and so we need to use the second order solution in this case. We obtain:

$$
x_{(2)}(z)=x_{0}^{\prime} L \frac{\gamma_{i}}{\Delta \gamma} \ln u+x_{0}^{\prime} L \frac{\gamma_{i}}{\Delta \gamma}[(u+1) \ln u-2(u-1)] \frac{r_{0}}{\gamma_{i} G^{2}} \int_{0}^{\infty} \rho\left(z^{\prime}+z\right) W_{\perp}\left(z^{\prime}\right) d z^{\prime}
$$

At the end of the linac $(u=1+G L)$, Eq.(13) is clearer in the following form:

$$
\frac{x_{(2)}}{x_{0}^{\prime} L}=\frac{\gamma_{i}}{\Delta \gamma} \ln (1+G L)+\frac{\gamma_{i}}{\Delta \gamma}[(2+G L) \ln (1+G L)-2 G L] \frac{r_{0}}{\gamma_{i} G^{2}} \int_{0}^{\infty} \rho\left(z^{\prime}+z\right) W_{\perp}\left(z^{\prime}\right) d z^{\prime}
$$

which points out the displacement relative to an injection angle error per unit length, after each linac pass.

Figure 3 shows numerical results for our parameters. The behaviour is qualitatively similar to that of Fig. 1, as it is expected comparing Eqs.(7) and (14).

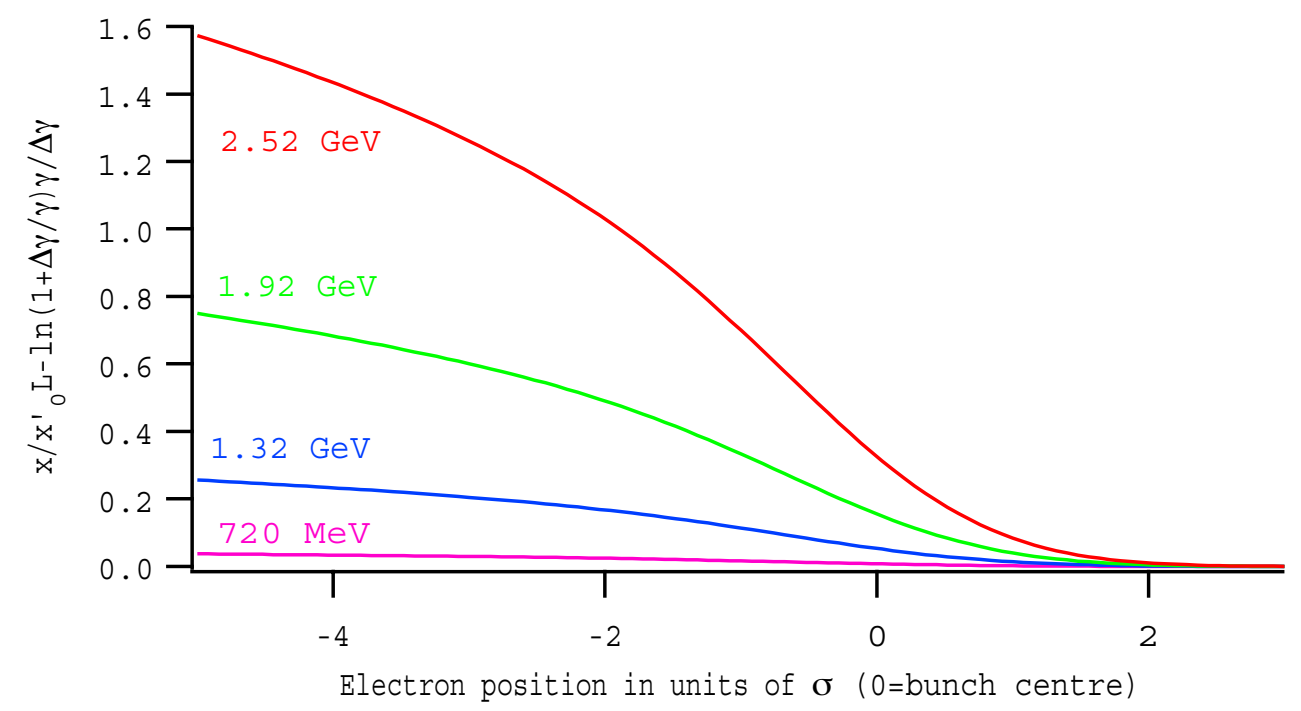

Figure 3. Electron displacement as a function of the position within the bunch (relative to the bunch head).

\section{VALIDITY LIMITS OF THE FIRST ORDER SOLUTION}

To check the validity of the calculations performed up to this point and in particular the implications of truncating to the first order term of the iterative solution, let's write the equation of motion for the most simple case, without acceleration and focussing: 


$$
\frac{\partial^{2} x(s, z)}{\delta s^{2}}=\frac{r_{0}}{\gamma_{0}} \int_{0}^{\infty} \rho\left(z+z^{\prime}\right) W_{\perp}\left(z^{\prime}\right) x\left(s, z+z^{\prime}\right) d z^{\prime}
$$

and the initial conditions

$$
x(s=0)=x_{0} \text { and } \frac{\partial x(s=0)}{\delta s}=0
$$

The iterative solution is in the form:

$$
\frac{\partial^{2} x_{(n)}}{\delta s^{2}}=\frac{r_{0}}{\gamma_{0}} \int_{0}^{\infty} \rho\left(z+z^{\prime}\right) W_{\perp}\left(z^{\prime}\right) x_{(n-1)}\left(s, z+z^{\prime}\right) d z^{\prime}
$$

with $x_{(0)}=x_{0}$.

From Eq.(17)

$$
x_{(n)}=x_{0}\left(\frac{r_{0}}{\gamma_{0}}\right)^{n} \frac{s^{2 n}}{(2 n) !} \mathfrak{\Im}_{n}(z)+x_{(n-1)}
$$

where

$$
\begin{aligned}
\mathfrak{I}_{n}(z)=\int_{0}^{\infty} \rho\left(z+z_{0}^{\prime}\right) W_{\perp}\left(z_{0}^{\prime}\right) \cdot & \\
& \quad \cdot \int_{0}^{\infty} \rho\left(z+z_{0}^{\prime}+z_{1}^{\prime}\right) W_{\perp}\left(z_{1}^{\prime}\right) \ldots \int_{0}^{\infty} \rho\left(z+z_{0}^{\prime}+z_{1}^{\prime}+\ldots+z_{n}^{\prime}\right) W_{\perp}\left(z_{n}^{\prime}\right) d z_{0}^{\prime} d z_{1}^{\prime} \ldots d z_{n}^{\prime}
\end{aligned}
$$

which means that

$$
x(s, z)=x_{0} \sum_{n=0}^{\infty}\left(\frac{r_{0}}{\gamma_{0}}\right)^{n} \frac{s^{2 n}}{(2 n) !} \Im_{n}(z)
$$

Under the hypothesis of uniform distribution bunch

$$
\rho(z)=\left\{\begin{array}{c}
N / \ell_{b} \text { for }|z|<\ell_{b} / 2 \\
0 \text { for }|z| \geq \ell_{b} / 2
\end{array}\right.
$$

and linear wake

$$
W_{\perp}(z)=W_{0}\left(1 / 2-z / \ell_{b}\right)
$$

Eq.(20) can be simplified and we have

$$
x(s, z)=x_{0} \sum_{n=0}^{\infty}\left(\frac{N W_{0} r_{0}}{\gamma_{0}}\right)^{n} \frac{s^{2 n}}{(2 n) !(2 n) !}
$$

where

$$
\xi=\left(1 / 2-z / \ell_{b}\right)
$$

Comparing Eq.(23) to the solution in the presence of strong focussing fields $\left(k_{\beta}=2 \pi / \lambda_{\beta}\right)[1]$ 


$$
x(s, L)=\operatorname{Re}\left[x_{0} e^{i k_{\beta} L} \sum_{n=0}^{\infty}\left(\frac{N W_{0} r_{0}}{\gamma_{0}}\right)^{n} \frac{\xi^{2 n}}{(2 n) !} \frac{L^{n}}{n !} \frac{1}{\left(i 2 k_{\beta}\right)^{n}}\right]
$$

we see that the only important difference is the absence of the oscillatory term driven by the betatron oscillations. If we instead compare to the case of an accelerated beam (Eq.(7)) we see that the effect of the acceleration appears in a weaker dependance on the coordinate $s$. For example, the first order term dependance is proportional to $s$ rather than to $s^{2}$, as in Eq.(18). All this points out that the worst scenario for the beam break up is a nonaccelerated beam, with no transverse focussing. Estimates made in this case are, therefore, conservative ones. From Eq.(23) we can also calculate the maximum length of the linac, for a given wakefunction, that it is still possible to analyze using the first order solution for the transverse displacement growth. To this end we calculate the linac length $L_{\max }$ at which, assuming that the energy doesn't change after injection, the additional displacement at the centre of the bunch $(z=0)$ is equal to the bunch initial displacement $x_{0}$ :

$$
\frac{x\left(s=L_{\max }, z=0\right)}{x_{0}}=\sum_{n=0}^{\infty}\left(\frac{N W_{0} r_{0}}{\gamma_{i}}\right)^{n} \frac{L_{\max }^{2 n}}{(2 n) !} \frac{(1 / 2)^{2 n}}{(2 n) !}=2
$$

which we can rewrite as:

$$
\sum_{n=0}^{\infty}\left\{\frac{\left[\sqrt{\frac{L_{\max }}{2} A^{1 / 2}}\right]^{2 n}}{(2 n) !}\right\}^{2}=\frac{1}{2}\left[I_{0}\left(\sqrt{2 L_{\max } A^{1 / 2}}\right)+J_{0}\left(\sqrt{2 L_{\max } A^{1 / 2}}\right)\right]=2
$$

where $A=N W_{0} r_{0} / \gamma_{i}$. Eq.(27) can be solved numerically yielding:

$$
\sqrt{2 L_{\max } A^{1 / 2}} \approx 2.81
$$

For the parameters in our case, we find from Eq.(28):

$$
L_{\max } \approx \sqrt{\frac{15.6 \gamma_{i}}{\mathrm{NW}_{0} r_{0}}} \approx 314 \mathrm{~m}
$$

This length is much longer than the effective linac length after 4 passes $(\sim 200 \mathrm{~m})$ thus validating all the approximations made in the previous sections.

\section{ASYMPTOTIC BEHAVIOUR}

It is interesting to study the dependance of the electron displacement on the beam energy and energy gain per pass. To this end, let's rewrite the average gradient $G$ as: 


$$
G=\left(\frac{\gamma_{i}+\Delta \gamma}{\gamma_{i}}-1\right) / L=\frac{\Delta \gamma}{\gamma_{i}} L^{-1}
$$

where $\Delta \gamma=\gamma_{f}-\gamma_{i}$ is the energy gained in a single pass.

We also can say that

$$
A_{0} \propto \frac{1}{\gamma_{i} G^{2}}=\frac{\gamma_{i}}{\Delta \gamma^{2}} L^{2} \text { and } A_{1} \propto\left(\frac{1}{\gamma_{i} G^{2}}\right)^{2}=\left(\frac{\gamma_{i}}{\Delta \gamma^{2}}\right)^{2} L^{4}
$$

We can recognize two different regimes depending on whether $G L<<1$ or $G L \gg>1$ which in turn implies $\Delta \gamma<<\gamma_{i}$ or $\Delta \gamma>>\gamma_{i}$. In general, we can say that the latter condition is verified in the first pass, while later passes satisfy the former.

The normalized displacement in Eq.(7) is therefore proportional to:

$$
\frac{x_{(1)}}{x_{0}}-1 \propto\left\{\begin{array}{l}
\frac{L^{2}}{2 \gamma_{i}} \text { for } \Delta \gamma \ll \gamma_{i} \\
\frac{L^{2}}{\Delta \gamma} \text { for } \Delta \gamma>>\gamma_{i}
\end{array}\right.
$$

The result in Eq.(32) is an interesting one since it tells us that in the first pass, which we already saw is the most critical one, the transverse displacement is independent on the injection energy. Furthermore, high energy gains per pass are better from this point of view, if obtained without increasing the linac length.

The second order approximation (Eq.(11)) adds to Eq.(32) a new term:

$$
\frac{x_{(2)}}{x_{0}}-1 \propto\left\{\begin{array}{l}
\frac{L^{2}}{2 \gamma_{i}}\left(1+\frac{C_{2}}{C_{1}} \frac{L^{2}}{12 \gamma_{i}}\right) \text { for } \Delta \gamma \ll \gamma_{i} \\
\frac{L^{2}}{\Delta \gamma}\left(1+\frac{C_{2}}{C_{1}} \frac{L^{2}}{4 \Delta \gamma}\right) \text { for } \Delta \gamma>>\gamma_{i}
\end{array}\right.
$$

where we have defined the new constants (with respect to energy and linac length) $C_{1}=r_{0} \int_{0}^{\infty} \rho\left(z^{\prime}+z\right) W_{\perp}\left(z^{\prime}\right) d z^{\prime}$ and $C_{2}=r_{0}^{2} \int_{0}^{\infty} \rho\left(z^{\prime}+z\right) W_{\perp}\left(z^{\prime}\right) \int_{0}^{\infty} \rho\left(\zeta+z^{\prime}+z\right) W_{\perp}(\zeta) d \zeta d z^{\prime}$.

The corresponding expressions for Eqs.(12) and (14) are normalized to $x_{0}^{\prime} L$ (i.e. the drift due to the initial angle) and the term relative to the first order approximation doesn't depend on $L$. We have:

$$
\frac{x_{(2)}}{x_{0}^{\prime} L} \propto\left\{\begin{array}{c}
1+C_{1} \frac{L^{2}}{6 \gamma_{i}} \text { for } \Delta \gamma \ll \gamma_{i} \\
\frac{\ln \left(\Delta \gamma / \gamma_{i}\right)}{\Delta \gamma / \gamma_{i}}\left(1+C_{1} \frac{L^{2}}{\Delta \gamma_{i}}\right) \text { for } \Delta \gamma>>\gamma_{i}
\end{array}\right.
$$


From Eq.(40) one can see that for small accelerations there is a wakefield-dependent term in the transverse displacement which is proportional to the length of the linac squared and inversely proportional to the initial beam energy. For very high accelerating gradients the displacement tends to zero instead.

\section{MISALIGNMENT EFFECTS}

In the previous sections we have assumed that the accelerator structure is perfectly aligned and the wake field is produced as a consequence of beam injection with a displacement, or angle, error. In this section we study the effect of misalignments of the RF cavities and cryomodules on the transverse dynamics.

To begin with, let's just consider errors in the cavities alignment. Equation (3), which gives the first order approximation for the transverse displacement, has to be only slightly modified to take this into account:

$$
\frac{d^{2} x_{(1)}}{d u^{2}}+\frac{1}{u} \frac{d x_{(1)}}{d u}=\frac{r_{0}}{\gamma_{i} G^{2} u} \int_{z}^{\infty} \rho\left(z^{\prime}\right) W_{\perp}\left(z^{\prime}-z\right)\left[x_{0}+d_{c}(u)\right] d z^{\prime}
$$

where the function $d_{c}(u)$ describes the misalignment. Considering only alignment offsets, we can write:

$$
d_{c}(u)=\sum_{i=0}^{N_{c a v}-1} d_{c i} \operatorname{rect}_{\operatorname{Cav}}\left[\frac{u}{G}-\frac{1-(1+1 / 2) G L_{c a v}}{G}\right]
$$

$N_{c a v}$ is the number of RF cavities and $L_{c a v}$ their length. For simplicity we can assume $L_{c a v}=L / N_{c a v} . d_{c i}$ is the displacement, or alignment error, of the $i$-th cavity and

$$
\operatorname{rect}_{C a v}(u)=\left\{\begin{array}{l}
0 \text { for } u<-L_{c a v} / 2 \\
1 \text { for }-L_{c a v} \leq u \leq L_{c a v} / 2 \\
0 \text { for } u>L_{c a v} / 2
\end{array}\right.
$$

Solving Eq.(41) we obtain

$$
x_{(1)}(s, z)=x_{0}+A_{0}(z)[G s-\ln (1+G s)]+x_{0}^{\prime} \ln (1+G s)+\frac{A_{0}(z)}{x_{0}} \sum_{i=0}^{N_{c a v}-1} d_{c i} F_{i}(s)
$$

where 


$$
\mathcal{F}_{i}(s)=\left\{\begin{array}{c}
0 \text { for } s<i L_{c a v} \\
G s-\left(1+i G L_{c a v}\right)\left[\ln \left(\frac{1+G s}{1+i G L_{c a v}}\right)\right]-i G L_{c a v} \text { for } i L_{c a v} \leq s \leq(i+1) L_{c a v} \\
G L_{c a v} \ln \left[1+\frac{1+G s}{1+(i+1) G L_{c a v}}\right]+ \\
-\left(1+i G L_{c a v}\right) \ln \left[\frac{1+(i+1) G L_{c a v}}{1+i G L_{c a v}}\right] \text { for } s>(i+1) L_{c a v}
\end{array}\right.
$$

From Eq.(44) we can see that the effect of cavity misalignments is to introduce an additional displacement that, in first approximation, does not depend on the injection coordinates $x_{0}$ and $x_{0}$ '. It's rms value can be calculated, knowing the misalignment rms value.

Choosing $x_{0}=x_{0}{ }^{\prime}=0$, for example, we find:

$$
\left\langle x^{2}\right\rangle^{1 / 2}=\frac{A_{0}(z)}{x_{0}} \sqrt{\left.\sum_{i=0}^{N_{c a v}-1} \mathcal{F}_{i}^{2}(s) \backslash d_{c}^{2}\right\rangle^{1 / 2}}
$$

In calculating Eq.(46), we have assumed no correlation between the alignment errors and this is strictly valid only when analyzing a single pass.

We can also account for the misalignments between the cryomodules in the same way. Equation (41) becomes

$$
\frac{d^{2} x_{(1)}}{d u^{2}}+\frac{1}{u} \frac{d x_{(1)}}{d u}=\frac{r_{0}}{\gamma_{i} G^{2} u} \int_{z}^{\infty} \rho\left(z^{\prime}\right) W_{\perp}\left(z^{\prime}-z\right)\left[x_{0}+d_{c}(u)+d_{m}(u)\right] d z^{\prime}
$$

We find equations analogous to Eqs.(21-25) with only $N_{c a v}$ and $L_{c a v}$ replaced by $N_{\text {mod }}$ and $L_{\text {mod }}$. Taking into account the two alignment errors, Eq.(46) becomes

$$
\left\langle x^{2}\right\rangle^{1 / 2}=\frac{A_{0}(z)}{x_{0}} \sqrt{\sum_{i=0}^{N_{c a v}-1} \mathcal{F}_{i}^{2}(s)\left\langle d_{c}^{2}\right\rangle+\sum_{i=0}^{N_{m d}-1} \mathcal{G}_{i}^{2}(s)\left\langle d_{m}^{2}\right\rangle}
$$

where 


$$
\mathcal{G}_{i}(s)=\left\{\begin{array}{l}
0 \text { for } s<i L_{\text {mod }} \\
G s-\left(1+i G L_{\text {mod }}\right)\left[\ln \left(\frac{1+G s}{1+i G L_{\text {mod }}}\right)\right]-i G L_{\text {mod }} \text { for } i L_{\text {mod }} \leq s \leq(i+1) L_{\text {mod }} \\
G L_{\text {mod }} \ln \left[1+\frac{1+G s}{1+(i+1) G L_{\text {mod }}}\right]+ \\
-\left(1+i G L_{\text {mod }}\right) \ln \left[\frac{1+(i+1) G L_{\text {mod }}}{1+i G L_{\text {mod }}}\right] \text { for } s>(i+1) L_{\text {mod }}
\end{array}\right.
$$

Assuming $L_{\text {mod }}=L / N_{\text {mod }}$, we obtain for the first pass in the main linac, at the bunch centre:

$$
\left\langle x^{2}(z=0)\right\rangle^{1 / 2} \approx 0.02 \sqrt{\left\langle d_{c}^{2}\right\rangle+7.7\left\langle d_{m}^{2}\right\rangle}
$$

for our parameters, 500 and $150 \mu \mathrm{m}$ [4-5] rms misalignments for the RF cavities and cryomodules respectively, Eq.(50) yields a value of about $10 \mu \mathrm{m}$ for the rms transverse displacement of the electrons at the bunch centre after the first linac pass.

In Fig.4 we compare Eq.(50) with the tracking code output for several other misalignments values, finding a fairly good agreement.

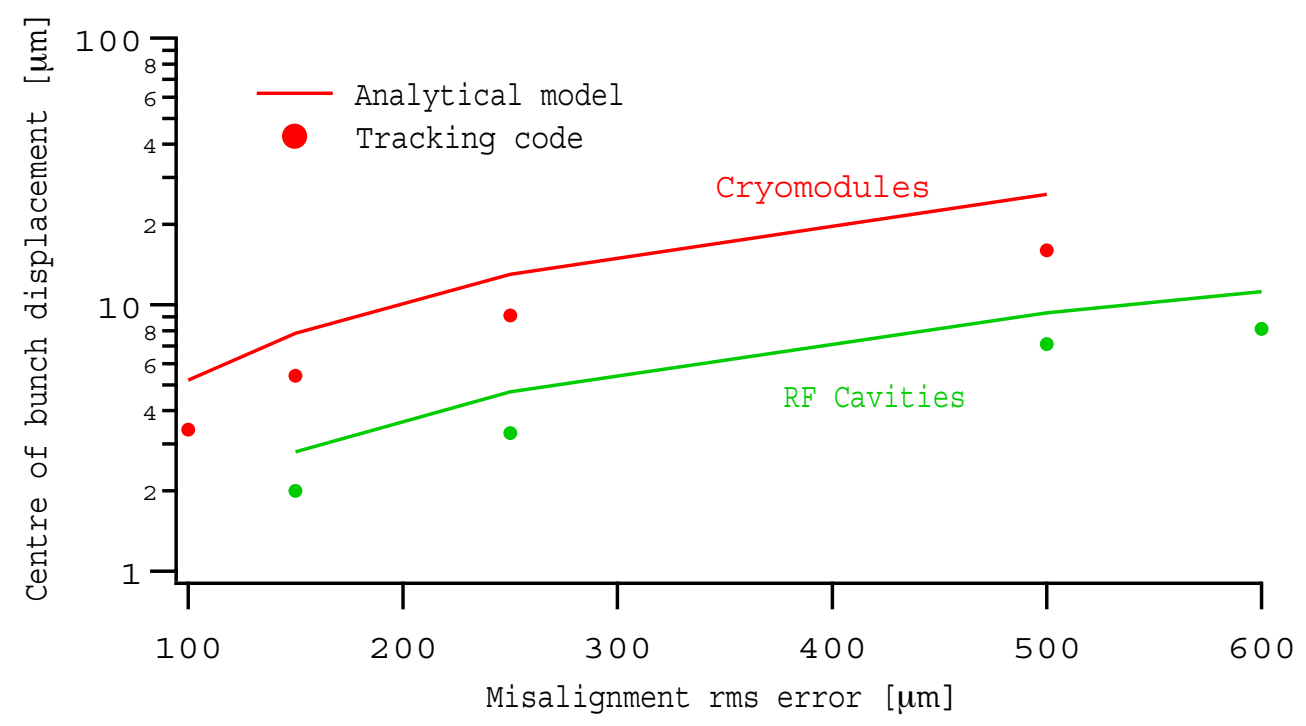

Figure 4. RMS value of the transverse displacement at the bunch centre $(z=0)$ as a function of RF cavities and cryomodules misalignments. 
A direct consequence of Eqs.(44) and (47) is that it is possible to cancel the effect of misalignments, at the linac exit, by choosing an opportune value for the initial displacement:

$$
x_{0}=-\sqrt{\frac{\left\langle d_{c}{ }^{2}\right\rangle \sum_{i=0}^{N_{c a v}-1} \mathcal{F}_{i}^{2}(L)+\left\langle d_{m}^{2}\right\rangle \sum_{i=0}^{N_{\text {mod }}-1} \mathcal{G}_{i}^{2}(L)}{G L-\ln (1+G L)}}
$$

This value can only be measured, after the linac has been assembled and aligned, by direct measurements of the beam profile. For our machine parameters, we expect the required offset to be in the range of $50 \div 100 \mu \mathrm{m}$.

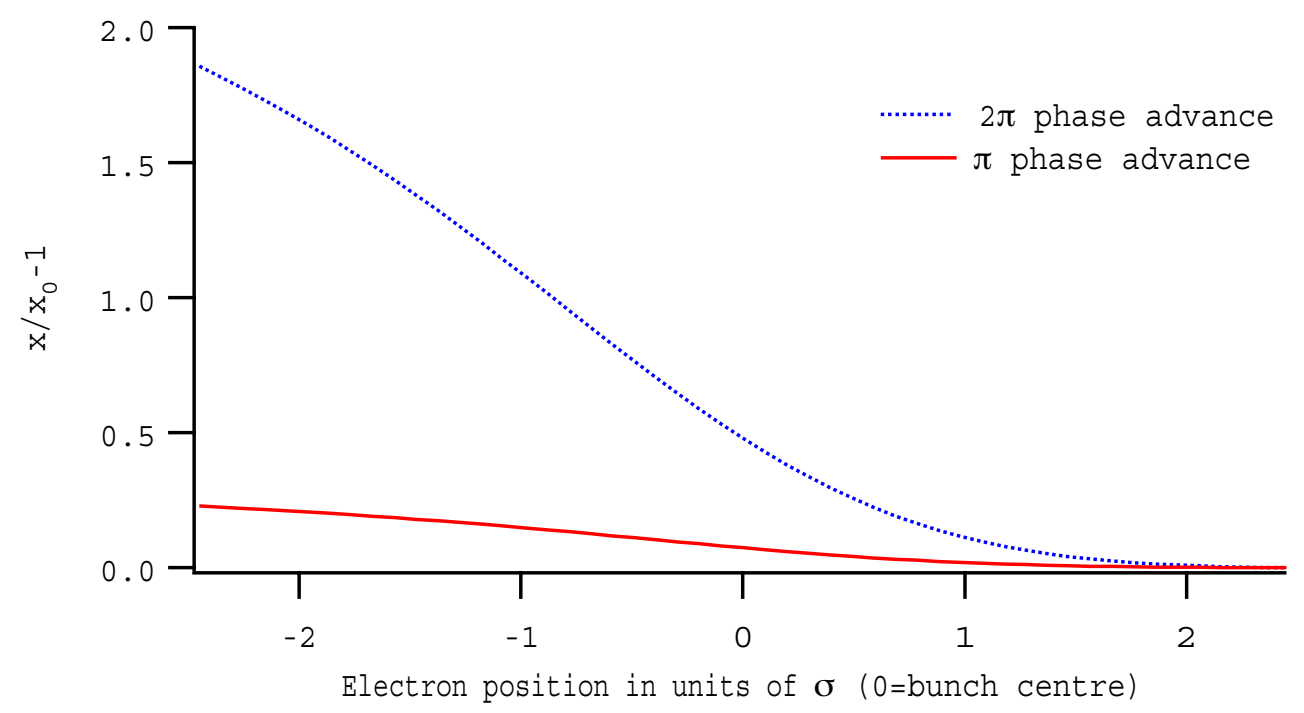

Figure 5. Electron displacement for zero betatron phase advance in all arcs (dotted line) and when the first arc has a $\pi$ phase advance.

It is also possible to design the the arcs in such a way that the subsequent linac passes will have a canceling effect on the displacement introduced in the first pass. Figure 5 shows the result from the tracking code whith a betatron phase advance of $\pi$ in the first and $2 \pi$ in the subsequent arc as opposed to the case of $2 \pi$ phase advance in all four arcs. The curves reported were obtained running a numerical tracking code with a $500 \mu \mathrm{m}$ rms displacement error in the cavities and cryomodules and a $50 \mu \mathrm{rad}$ exit-entrance tilt error in the cryomodules. The improvement can be explained by the fact that changing the sign of the betatron phase along the bunch after the first turn effectively causes the wakefield to reduce the transverse displacement in later passes. 


\section{PRE-ACCELERATOR}

Before being injected in the main linac, the electron bunch is accelerated from 10 to 120 $\mathrm{MeV}$ in a pre-accelerator section. This pre-accelerator is composed of a single cryomodule of the same kind as in the main linac. Therefore we will assume that the wake fields are the same and use the same formulas already derived for the main linac with just different length $(8.0 \mathrm{~m})$, injection energy and energy gain.

An important difference with the main linac is that, due to the lower energy, the bunch is subject to the focussing effect from the RF field [6], following its injection in the preaccelerator.

This effect is included in Eq.(1), when there are no other focussing elements, by writing:

$$
k^{2}(s)=\frac{1}{2}\left(\frac{r_{0} G}{e \gamma}\right)^{2}
$$

where $G \approx 14 \mathrm{MV} / \mathrm{m}$ is the average accelerating gradient in the pre-accelerator.

Given the high relative energy change (12 times, from injection to extraction) the betatron wavelength changes from $4.83 \mathrm{~m}$ at injection to $31.4 \mathrm{~m}$ at the end of the pre accelerator. Rather than trying to solve directly Eq.(1), we can just check that in the absence of focussing the wake field effect is still below the maximum we can tolerate. This will be a conservative estimate since focussing can only help control the BBU growth. Furthermore, we assume a constant energy equal to the value at injection, effectively putting ourselves in the worst possible conditions.

From Eq.(7) it is readily seen that the bunch centre transverse displacement at the end of the linac, for a $1.5 \mathrm{~mm}$ bunch, is

$$
\frac{x(z=0)}{x_{0}} \approx 2.310^{-3}
$$

Obviously, this is a small effect that can be ignored.

\section{CONCLUSIONS}

In this paper we describe a study of a single bunch beam break-up instability for recirculating linac based on the Tesla superconducting RF cavities.

We develop the analytical model of the phenomena suitable for the case under study and use it to obtain closed form solutions. We also show the validity boundaries of the model.

Our study suggests that mechanical misalignments of RF cavities and cryomodules are expected to be the strongest source of the instability. We propose to use a particular pattern 
in the betatron phase advance in successive beam passes through the linac to minimize the beam break up growth.

We also show that it is possible to eliminate the instability in all practical cases of alignment errors by injecting the beam with an orbit offset to be determined by beam-based maesurements.

All analytical results were tested with a specially developed tracking code.

\section{APPENDIX A}

The recirculated linac parameters used in our calculations are the following:

- Main linac length $L=48.8 \mathrm{~m}$.

- Injected energy $\gamma_{i}=235\left(1^{\text {st }}\right.$ pass, $\left.120 \mathrm{MeV}\right)$

- $1412\left(2^{\text {nd }}\right.$ pass, $\left.720 \mathrm{MeV}\right)$

- $2588\left(3^{\text {rd }}\right.$ pass, $\left.1320 \mathrm{MeV}\right)$

- $3765\left(4^{\text {th }}\right.$ pass, $\left.1920 \mathrm{MeV}\right)$

- Energy gain per pass $\Delta \gamma=1176(600 \mathrm{MeV})$

- Number of electrons per bunch ( $1 \mathrm{nC}): N=6.2510^{9}$

- Bunch length (used to calculate $\left.W_{\perp}(z)\right) \sigma=1.5 \mathrm{~mm}$.

- $\quad$ Number of RF cavities $N_{c a v}=32$

- $\quad$ Number of cryomodules $N_{\text {mod }}=4$

\section{APPENDIX B}

The tracking code is written as a Mathematica notebook. The bunch is divided longitudinally in a number of slices, or macroparticles, that can be choosen by the user. A gaussian or a uniform charge distribution can also be selected.

The linac is modeled as a series of $32 \mathrm{RF}$ cavities, grouped in 4 cryomodules and separated by drift spaces.

The transverse coordinate $x_{i}$ of each slice at the exit of the $i$-th cavity is calculated as a function of the displacement $x_{i-1}$ and angle $x_{i-1}^{\prime}$ at the exit of the preceding cavity:

$$
x_{i}=x_{i-1}+x_{i-1}^{\prime} L_{d r i f t}+x_{i-1}^{\prime} L_{c a v}+\Delta x_{i-1}^{\prime} \frac{L_{c a v}}{2}
$$


The additional transverse kick $\Delta x_{i}^{\prime}$ represents the cumulative effect of the wakefield induced by the upstream macroparticles. The wakefields are calculated using the analytical formula from [3-4].

The macroparticles angle also changes along the linac:

$$
x_{i}^{\prime}=x_{i-1}^{\prime}+\Delta x_{i-1}^{\prime}-\frac{x_{i-1}}{2 L_{c a v}}\left(\frac{\Delta \gamma}{\gamma_{i-1}}\right)^{2}
$$

where the effect of the RF focussing is taken into account by the last term in Eq.(B.2).

It is also possible to introduce misalignments between cavities and cryomodules and cryomodules tilts. Again, the user can choose amongst different statistical distribution for these errors and determine the most probable values for the emittance increase, performing simulations using many sets of randomly generated alignment errors.

Finally, the user can arbitrarily select the betatron phase advance between each linac pass.

\section{REFERENCES}

[1] J. Corlett, et. al., LBNL-48171 (2001).

[2] A. Chao, B. Richter, C.-Y. Yao, SLAC-PUB-2498 (1980).

[3] A. Mosnier, TESLA 93-11 (1993).

[4] TESLA Technical Design Report (2001).

[5] ALS Alignment Group, private communication.

[6] S. Hartman and J. Rosenzweig, Phys. Rev. E 47, 2031 (1993). 